\title{
Management of Solid-pseudopapillary Neoplasms of the Pancreas: a Comparison with Standard Pancreatic Neoplasms
}

\author{
S. M. M. de Castro, MD, ${ }^{1}$ D. Singhal, MD, ${ }^{1}$ D. C. Aronson, $M D,{ }^{2}$ O. R. C. Busch, MD, ${ }^{1}$ \\ T. M. van Gulik, MD, ${ }^{1}$ H. Obertop, MD, ${ }^{1}$ D. J. Gouma, MD $^{1}$ \\ ${ }^{1}$ Department of Surgery, Academic Medical Center, Meibergdreef 9, 1105 AZ, Amsterdam, The Netherlands \\ ${ }^{2}$ Department of Pediatric Surgery, Academic Medical Center, Meibergdreef 9, 1105 AZ, Amsterdam, The Netherlands
}

\begin{abstract}
Background: Solid-pseudopapillary neoplasms (SPNs) of the pancreas are increasingly diagnosed, but the exact surgical management in terms of extent of the resection is not well defined. Materials and Methods: Patients operated on in our hospital between January 1993 and March 2005 formed the study groups.

Results: From 659 consecutive resections for pancreatic neoplasms, 12 female patients (1.8\%) with a median age of 21 years who underwent resection for (SPN) are compared with the remaining 647 pancreatic resection patients. Jaundice (SPN 0 versus PR 73\%, $p<0.001$ ) and weight loss (SPN 0 versus PR 49\%, $p=0.001$ ) occurred significantly less often. Neoplasms were distributed equally among the pancreatic head (SPN 5 out of 12 patients versus PR $88 \%$, $p<0.001$ ) and corpus/tail (SPN 6 out of 12 patients versus PR $8 \%, p<0.001$ ). The operative time was significantly shorter (SPN 233 min versus PR $280 \mathrm{~min}, p=0.012$ ), and there were significantly fewer complications (SPN 1 of 12 patients versus PR $48 \%, p=0.007$ ). The mortality was not different (SPN 0 versus PR $1.6 \%, p=1.000$ ), and the hospital stay was significantly shorter (SPN 9 days versus PR 15 days, $p=0.012$ ). The median size of the neoplasms was significantly larger (SPN $6.9 \mathrm{~cm}$ versus PR $2.5 \mathrm{~cm}$ ). The median number of lymph nodes harvested was significantly fewer (SPN 1 versus PR 6, $p=0.001$ ), and lymph node metastases occurred significantly less often (SPN 0 versus PR $64 \%, p<0.001$ ). The 5 -year survival of SPN patients was $100 \%$ and is significantly better compared with survival of patients with pancreatic adenocarcinoma $(12 \%, p<0.001)$ and ampulla of Vater adenocarcinoma $(22 \%, p=0.005)$.

Conclusions: Patients with solid-pseudopapillary neoplasms of the pancreas present differently and the course of the disease is more benign. These patients can be adequately managed by pylorus-preserving pancreatoduodenectomy or spleen-preserving distal pancreatectomy with excellent early and long-term results.
\end{abstract}

S olid-pseudopapillary neoplasm (SPN) of the pancreas was first reported by Frantz in $1959 .{ }^{1}$ Further specification by Hamoudi led to its acceptance as a separate clinicopathological entity. ${ }^{2}$ There have been controversies regarding the terminology, and it has been

\footnotetext{
Correspondence to: D. J. Gouma, MD, e-mail: d.j.gouma@amc. uva.nl
}

concluded that the semantically correct description is solid-pseudopapillary neoplasm. ${ }^{3}$ The neoplasm remained essentially unrecognized up to the early 1990s, and fewer than 300 cases were reported up until 1995. ${ }^{4}$ However, with increased awareness among surgeons, radiologists, and pathologists and the widespread availability of new high-quality imaging systems, the number 
of reported cases has doubled in less than a decade to approximately 600 annually. ${ }^{5}$ Solid-pseudopapillary neoplasms make-up approximately $1 \%-2 \%$ of all pancreatic neoplasms. ${ }^{6,7}$

The cumulative experience to date has provided interesting insights into this disease. Most patients (up to $90 \%$ ) are female and usually in the second or third decade of life. ${ }^{4,8-11}$ The neoplasms have a low malignant potential, with a reported incidence of malignant transformation of around $15 \% .{ }^{11}$ The biological behavior in terms of metastasis or local invasion is the most accepted criterion for malignant transformation. ${ }^{4,9}$ This low level of aggressiveness corresponds to the high cure rate. ${ }^{11}$ Malignant SPN is still compatible with long-term survival in patients undergoing radical resection of the neoplasm. ${ }^{6}$

The surgical management of these neoplasms is still not well defined. ${ }^{4,6,8}$ There are no definite recommendations in the literature regarding to the extent of resection of the primary neoplasm and management of metastasis. These features of the disease assume increased importance because more patients with these neoplasms are being diagnosed preoperatively and are advised to undergo pancreatic resection.

The aim of the present study was therefore to analyze the clinical presentation, diagnostic work-up, management, and outcome of patients with SPNs of the pancreas, and to compare the results with patients who undergo resection for all other pancreatic neoplasms.

\section{PATIENTS AND METHODS}

The prospective pancreatic database (January 1993March 2005) of the department of Surgery and Pediatric Surgery of the Academic Medical Center, Amsterdam, was used to identify patients with SPNs of the pancreas managed in this period. The patients with pathologically proven SPN formed the study group and were compared to a pancreatic resection group (PR) consisting of all other patients who underwent resection for pancreatic neoplasms. Procedures included pancreatoduodenectomy, central pancreatectomy, and distal pancreatectomy. Enucleations for neuroendocrine neoplasms were excluded.

The patient records were used to collect demographic, clinical, operative, and pathologic data. The preoperative data included age at presentation, sex, symptoms, imaging studies, and clinical diagnosis. Weight loss was defined as a loss of $10 \%$ of body weight during the previous year. The patients in whom a preoperative diagnosis of SPN of the pancreas was made were studied separately to identify the clinical and imaging character- istics that led to the diagnosis. The surgical parameters analyzed included the location of the neoplasm, evidence of malignancy (metastasis or local invasion), and the type of procedure performed.

Postoperative complications and length of hospital stay were recorded. The definitions of complications used for the present study have been reported previously. ${ }^{12,13}$ In short, pancreatic fistula was defined as abdominal drain fluid with amylase levels three times the normal serum levels. Delayed gastric emptying after PPPD was defined as either the need for nasogastric intubation for 10 days or more or the inability to tolerate regular food before or on postoperative day (POD) 14. In-hospital mortality was defined as death hospitalization stay or during readmission within 30 days.

The histopathological data analyzed comprised the size of the neoplasm; radicality of resection; involvement of lymph nodes and histological features suggestive of malignancy, perineural or angioinvasion, or invasion into the surrounding tissue; and immunohistochemical staining. A curative resection (R0) was characterized by a specimen with no gross tumor mass remaining at the operation site or in other organs and microscopic clear resection margins. An R1 resection was defined as microscopic involvement of the resection margin, and R2 was defined by microscopically confirmed macroscopic tumor remaining at the operation site. Only lymph nodes surrounding the pancreas anteriorly and posteriorly, in the hepatoduodenal ligament, and right of the common hepatic artery and superior mesenteric vein were removed. The follow-up data was recorded through review of the hospital records, the outpatient visits, and telephonic interview.

Data analyses were performed using SPSS ${ }^{\circledR}$ software (SPSS, Chicago, Illinois, USA). A value of $p<0.05$ was considered statistically significant. Univariate analysis was carried out with Pearson's $\chi^{2}$ test to determine which variables were statistically significant. Fisher's exact test was used when a table had a cell with an expected frequency of less than 5 .

\section{RESULTS}

Histopathological features that confirmed the diagnosis of SPN of the pancreas were found in 12 of the 659 consecutive patients $(1.8 \%)$ who underwent pancreatic resection during the study period. All 12 patients were female with a median age of 21 (range: 13-55) years (Table 1). Abdominal pain was the most common presenting symptom, and it occurred in 8 patients (67\%). 
Table 1.

Characteristics of patients who underwent pancreatic resection ${ }^{1}$

\begin{tabular}{|c|c|c|c|}
\hline & $\begin{array}{l}\text { Pancreatic resection for neoplasms } \\
\qquad(n=647)\end{array}$ & $\begin{array}{c}\text { SPN } \\
(n=12)\end{array}$ & $p$ Value \\
\hline Age (median, range) & $63(28-88)$ & $21(13-55)$ & $<0.001^{4}$ \\
\hline Gender (male:female) & $350: 297$ & $0: 12$ & $0.002^{2}$ \\
\hline \multicolumn{4}{|l|}{ Symptoms } \\
\hline Jaundice $(\%)$ & $474(73)$ & 0 & $<0.001^{3}$ \\
\hline Weight loss $(\%)$ & $317(49)$ & 0 & $0.001^{2}$ \\
\hline Pain $(\%)$ & $266(41)$ & 8 & $0.084^{3}$ \\
\hline Surgery & & & \\
\hline PD or PPPD (\%) & $570(88)$ & 5 & $<0.001^{3}$ \\
\hline Tail resection $(\%)$ & $50(8)$ & 6 & $<0.001^{3}$ \\
\hline Corpus resection (\%) & 19 (3) & 1 & $0.311^{3}$ \\
\hline Complications (\%) & $310(48)$ & 1 & 0.007 \\
\hline Operative time (median, range) & $280(40-685)$ & $223(120-390)$ & $0.012^{4}$ \\
\hline Mortality (\%) & $10(1.6)$ & 0 & $1.000^{3}$ \\
\hline Postoperative hospital stay in days (median, range) & $15(6-222)$ & $9(6-18)$ & $<0.001^{4}$ \\
\hline
\end{tabular}

PD: pancreatoduodenectomy; PPPD: pylorus preserving pancreatoduodenectomy; SPN: solid-pseudopapillary neoplasm.

${ }^{1}$ Numbers in parentheses are percentages.

${ }^{2} \chi^{2}$ test.

${ }^{3}$ Fisher's exact test.

${ }^{4}$ Mann-Whitney U-test.

None of the patients presented with jaundice (SPN 0 versus PR 73\%, $p<0.001$ ), and weight loss (SPN 0 versus PR $49 \%, p=0.001$ ) occurred significantly less often.

Contrast-enhanced computed tomography (CT) scan of the abdomen was performed in all patients. The CT scan findings suggestive of the diagnosis of SPN were a large heterogeneous mass with solid and cystic components, as well as areas of hyperattenuation representing intramural hemorrhage.

Three patients underwent preoperative fine-needle aspiration cytology (FNAC). The FNAC was inconclusive in the one patient and confirmed SPN in two. The preoperative diagnosis in the first four consecutive patients was serous cystic neoplasm after CT scan, all diagnosed before 2000. After 2000, 6 patients were preoperatively diagnosed as having a SPN, and one 54-year-old patient was thought to have a serous cystic neoplasm. The lesion was located in the head of the pancreas in five patients. All of these patients underwent a pylorus preserving pancreatoduodenectomy. The remainder of the patients had a distal neoplasm (corpus or tail).

Distal pancreatectomy was performed in six patients. The spleen was preserved in two out of six patients who underwent distal pancreatectomy. Spleen preservation was not possible in one patient because of vascular involvement (splenic artery and vein), one had hilar involvement, one had splenomegaly, and one was managed in the early years of the series. Liver metastases in the right liver lobe were detected in one patient and a right hemihepatectomy was performed 5 months later. A central pancreatectomy was performed in one patient. Neoplasms occurred less often in the pancreatic head (SPN in 5 of 12 patients versus PR $88 \%, p<0.001$ ) and more often in the corpus/tail (SPN 6 of 12 patients versus PR $8 \%, p<0.001)$. Central neoplasms suitable for central pancreatectomy (SPN 1 of 12 patients versus PR 3\%, $p=0.311$ ) did not differ between groups. The operative time was significantly shorter (SPN 233 min versus PR $280 \min , p=0.012$ ).

One patient developed a postoperative complication and no in-hospital mortality occurred. One patient developed cholangitis postoperatively which was successfully managed (conservatively) with antibiotics. There were significantly less postoperative complications (SPN 1 patient of 12 versus PR $48 \%, p=0.007$ ) and the postoperative hospital stay was significantly shorter (SPN 9 days versus PR 15 days, $p=0.012$ ). The in-hospital mortality was comparable (SPN 0 versus PR $1.6 \%, p=1.000$ ).

At pathology (Table 2), the median size of the neoplasms was 6.9 (range: 3-16) $\mathrm{cm}$ and all neoplasms were well circumscribed with no local infiltration on gross examination. At histopathologic examination, all resections were radical $(\mathrm{R} 0)$. No differences could be recognized in the microscopic picture between 11 patients with benign SPN and the one with metastatic SPN. Lymph node metastasis was not found in any of the patients (including the metastatic SPN). The median neoplasm size was significantly larger (SPN $6.9 \mathrm{~cm}$ versus PR $2.5 \mathrm{~cm}, p<0.001$ ) and positive lymph nodes (SPN 0 versus PR 64\%, $p<0.001$ ) occurred significantly less often. Immunohistochemical 
Table 2.

Pathology findings for patients undergoing pancreatic resection versus those undergoing solid-pseudopapillary neoplasm ${ }^{1}$

\begin{tabular}{lllr}
\hline & \multicolumn{1}{c}{ Pancreatic resection for neoplasms } & \multicolumn{1}{c}{ SPN } & $p$ Value \\
& \multicolumn{1}{c}{$(n=647)$} & $6.9(3.0-16.0)$ & $<0.001^{3}$ \\
\hline Median neoplasm size in cm (range) & $2.5(0.3-11.0)$ & $1^{2}$ & $<0.001^{4}$ \\
Malignancy & $525(81)$ & 12 & $0.005^{4}$ \\
R0 resection & $395(61)$ & $1(0-11)$ & $0.001^{6}$ \\
Median no. of lymph nodes harvested (range) & $6(0-29)^{5}$ & 0 & $<0.001^{6}$ \\
\hline Positive lymph nodes & $336(52)$ & 0
\end{tabular}

${ }^{1}$ Numbers in parentheses are percentages.

${ }^{2}$ One patent had metastatic lesions $(0.5 \mathrm{~cm}$ and $1.5 \mathrm{~cm})$, both in segment 8 .

${ }^{3}$ Mann-Whitney U-test.

${ }^{4}$ Fisher's exact test.

${ }^{6}$ Including the specimens of patients with benign neoplasms.

${ }^{6} \chi^{2}$ test.

Table 3.

Immunohistochemical staining of solid-pseudopapillary neoplasms of the pancreas $(n=11)$

\begin{tabular}{lll}
\hline \multicolumn{1}{c}{ Stain } & Positive (\%) & Negative (\%) \\
\hline N-CAM/CD56 & 11 & 0 \\
Chromogranin A & 2 & 9 \\
Synaptophysin & 2 & 9 \\
Vimentine & 11 & 0 \\
Cytokeratine & 1 & 10 \\
Neurospecific enulase & 11 & 0 \\
CD10 & 11 & 0 \\
Alfa-1-antitrypsin & 9 & 2 \\
B-catenin & 11 & 0 \\
CAM 5.2 & 11 & 0 \\
\hline
\end{tabular}

staining (Table 3) was performed on 11 specimens and positive for CD56, vimentin and $\beta$-catenin in all patients, further confirming the diagnosis of SPN.

At a median follow-up of 3.8 years (range: 2-12.6 years) all SPN patients are alive and well. The survival is significantly better compared to pancreatic adenocarcinoma and ampulla of Vater adenocarcinoma, the two most common neoplasms in the pancreatic resection group (Fig. 1). In the present study the number of cases was equal to the number of significant variable found in univariate analysis, and therefore a multivariate analysis was not performed.

\section{DISCUSSION}

The present study reports the results of the surgical management of SPNs of the pancreas and found that patients generally present with abdominal pain. These patients have large neoplasms, which are equally distributed among proximal and distal pancreas. Pylorus- preserving pancreatectomy and distal pancreatectomy resulted in a microscopic $\mathrm{R} 0$ in all patients, with virtually no postoperative complications and no in-hospital mortality.

Contrast-enhanced CT scan of the abdomen with thin slices was the imaging modality used for the diagnosis of these neoplasms. An appropriate clinical setting (i.e., a young and female patient) together with the appearance of a heterogeneously enhancing solid-cystic neoplasm resulted in the preoperative diagnosis. There are studies that report that magnetic resonance imaging (MRI) scan may be superior for the diagnosis of SPN because it better depicts the neoplasm capsule and the intratumoral hemorrhage that is frequently associated with SPN. ${ }^{14,15}$ A CT scan or MRI scan should be conclusive so that FNAC is not necessary before surgery is planned, and furthermore resection is indicated in patients with a resectable pancreatic mass.

The experience with fine FNAC (percutaneous or EUS guided) for the diagnosis of SPN is limited. ${ }^{5,16}$ In the present study, after percutaneous FNAC in three patients, a definitive diagnosis of SPN was reached in two. A recent study reported a "preliminary" diagnosis of SPN in only one of six patients, the other diagnoses being lowgrade neoplasm in two and endocrine neoplasm in three. ${ }^{5}$ Fine-needle aspiration cytology therefore may be useful in obtaining the diagnosis, but it usually is not necessary.

Surgery for SPN of the pancreas is increasingly being performed because these neoplasms are now being diagnosed more frequently. ${ }^{5}$ This is reflected in the present study, where seven patients with this rare neoplasm were managed in the last 2 years of the studied period.

Preserving surgery for SPN of the pancreas is advocated by one other study in the literature. ${ }^{8}$ The role of more limited surgery in the form of neoplasm enucleation 


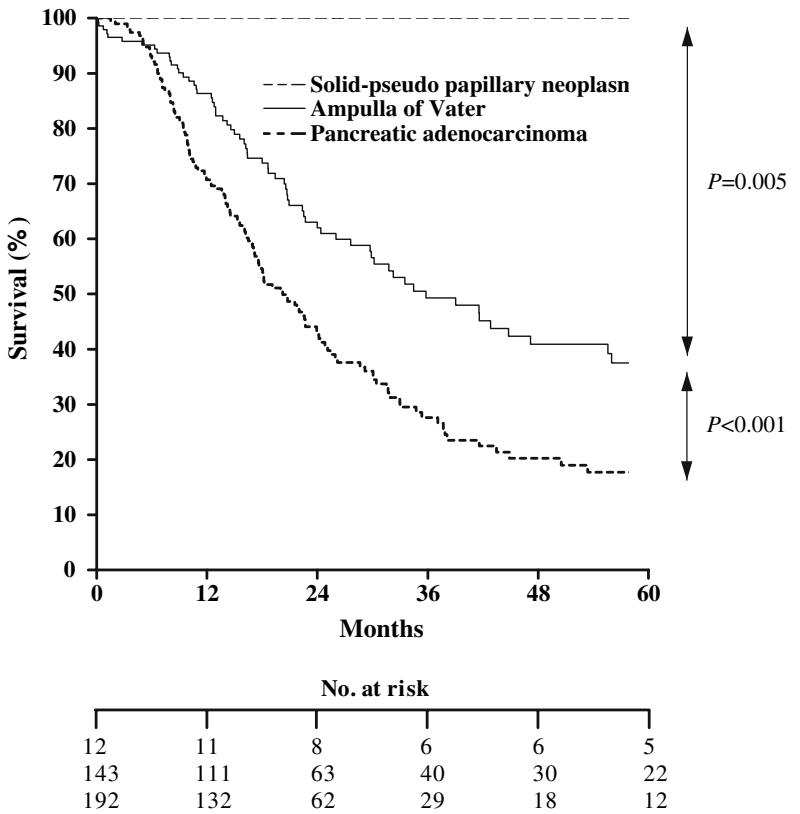

Figure 1. Survival after resection for SPN and the two most common pancreatic neoplasms.

is uncertain, and there are only case reports of management of SPN by this approach. ${ }^{9}$ Enucleation was not preformed in the present study because of the high risk of pancreatic fistula. ${ }^{17,18}$

Pylorus-preserving pancreatoduodenectomy and spleen-preserving distal pancreatic resections are now acceptable procedures. However, spleen preservation was possible in only two of six distal pancreatectomies performed in the present study. The spleen can be safely preserved without preservation of the splenic artery and vein. ${ }^{19}$ Spleen preservation may also become difficult because of the relatively large size of the neoplasms. The value of a central pancreatic resection is obviously that more pancreatic tissue is spared in these young patients. However, in view of the soft pancreas that is present in these patients with consequent higher chances of pancreaticoenteric anastomotic leak, it remains controversial. ${ }^{18}$ It may be argued that this type of surgery should be restricted to specialized centers with adequate experience.

The experience with the management of disseminated disease is very limited in the literature. Liver metastases are quite uncommon with SPN, and in many patients their removal results in long-term survival. ${ }^{6,7}$ Long-term survival has also been reported in patients whose liver metastases were not resected. ${ }^{20,22}$ In these reported series, two patients with liver metastasis had significant overall survival, with one alive at 11 years and the other alive with liver recurrence at 4 years.
There are no reports in the literature that describe the sensitivity to radiotherapy or chemotherapy, and such adjuvants probably have no effect on these neoplasms. One of the questions pertaining to the surgical management of SPN that remains unanswered is the extent of resection for liver metastases. The only such patient in the current series, managed by right hemihepatectomy over a decade ago, is currently disease free. However, keeping in mind the biological behavior of the neoplasm, a metastectomy with a $1-\mathrm{cm}$ margin would probably be sufficient. Similarly, peritoneal metastases are very rare, and there are reports of long-term survival after debulking. ${ }^{21}$ Ingrowth into adjacent organs like stomach or spleen would necessitate the excision of part of the involved organs. However, in patients where the involvement is extensive enough to preclude resection, extended survival is still possible. ${ }^{20}$

The incidence of lymph node metastasis is extremely rare. ${ }^{6}$ A review of 292 SPNs reported 43 as malignant $(15 \%)$, and of these, lymph nodes were involved in only five patients $(2 \%){ }^{4}$ Thus, a formal lymphadenectomy is not indicated in patients with (malignant) SPN and was not routinely performed in the present study.

In conclusion, patients with solid-pseudopapillary neoplasms of the pancreas present differently from patients with other pancreatic neoplasms. The present study suggests a standardized policy of resection for patients with SPN in view of the relatively benign behaviour of these neoplasms, the young age of these patients, and the complete alleviation of all symptoms after resection with no mortality. The patients with a lesion localized to the head of the pancreas should undergo PPPD, and lesions in the tail should be treated with a spleen-preserving distal pancreatectomy. Selected neoplasms of the body of the pancreas (small size) can be managed by corpus resection if experience is at hand.

\section{REFERENCES}

1. Frantz VK. (1959) Atlas of Tumor Pathology Armed Forces Institute of Pathology, Washington, DC.

2. Hamoudi AB, Misugi $K$, Grosfeld JL, et al. Papillary epithelial neoplasm of pancreas in a child. Report of a case with electron microscopy. Cancer 1970;26:1126-1134.

3. Sarr MG, Warshaw AL. Tumor versus neoplasm: Isn't it time to use the correct term-neoplasm? Surgery 2005; 137:297.

4. Mao C, Guvendi M, Domenico DR, et al. Papillary cystic and solid tumors of the pancreas: a pancreatic embryonic tumor? Studies of three cases and cumulative review of the world's literature. Surgery 1995;118:821-828. 
5. Bardales RH, Centeno B, Mallery JS, et al. Endoscopic ultrasound-guided fine-needle aspiration cytology diagnosis of solid-pseudopapillary tumor of the pancreas: a rare neoplasm of elusive origin but characteristic cytomorphologic features. Am J Clin Pathol 2004;121:654-662.

6. Martin RC, Klimstra DS, Brennan MF, et al. Solid-pseudopapillary tumor of the pancreas: a surgical enigma?. Ann Surg Oncol 2002;9:35-40.

7. Klimstra DS, Wenig BM, Heffess CS. Solid-pseudopapillary tumor of the pancreas: a typically cystic carcinoma of low malignant potential. Semin Diagn Pathol 2000;17:6680.

8. Panieri E, Krige JE, Bornman PC, et al. Operative management of papillary cystic neoplasms of the pancreas. $J$ Am Coll Surg 1998;186:319-324.

9. Lam KY, Lo CY, Fan ST. Pancreatic solid-cystic-papillary tumor: clinicopathologic features in eight patients from Hong Kong and review of the literature. World J Surg 1999; 23:1045-1050.

10. Jung SE, Kim DY, Park KW, et al. Solid and papillary epithelial neoplasm of the pancreas in children. World J Surg 1999;23:233-236.

11. Tipton SG, Smyrk TC, Sarr MG, et al. Malignant potential of solid pseudopapillary neoplasm of the pancreas. Br J Surg 2006;93:733-737.

12. Gouma DJ, van Geenen RC, van Gulik TM, et al. Rates of complications and death after pancreaticoduodenectomy: risk factors and the impact of hospital volume. Ann Surg 2000;232:786-795.

13. Berge Henegouwen MI, de Wit LT, van Gulik TM, et al. Incidence, risk factors, and treatment of pancreatic leakage after pancreaticoduodenectomy: drainage versus resection of the pancreatic remnant. J Am Coll Surg 1997;185:18-24.

14. Buetow PC, Buck JL, Pantongrag-Brown L, et al. Solid and papillary epithelial neoplasm of the pancreas: imagingpathologic correlation on 56 cases. Radiology 1996; 199:707-711.

15. Cantisani V, Mortele KJ, Levy A, et al. MR imaging features of solid pseudopapillary tumor of the pancreas in adult and pediatric patients. AJR Am J Roentgenol 2003;181:395401.

16. Mergener K, Detweiler SE, Traverso LW. Solid pseudopapillary tumor of the pancreas: diagnosis by EUS-guided fine-needle aspiration. Endoscopy 2003;35:1083-1084.

17. Rothmund M, Angelini L, Brunt LM, et al. Surgery for benign insulinoma: an international review. World J Surg 1990; 14:393-398.

18. Efron DT, Lillemoe KD, Cameron JL, et al. Central pancreatectomy with pancreaticogastrostomy for benign pancreatic pathology. J Gastrointest Surg 2004;8:532-538.

19. Warshaw AL. Conservation of the spleen with distal pancreatectomy. Arch Surg 1988;123:550-553.

20. Zinner MJ, Shurbaji MS, Cameron JL. Solid and papillary epithelial neoplasms of the pancreas. Surgery 1990; 108:475-480.

21. Rebhandl W, Felberbauer FX, Puig S, et al. Solid-pseudopapillary tumor of the pancreas (Frantz tumor) in children: report of four cases and review of the literature. J Surg Oncol 2001;76:289-296.

22. Nishihara K, Nagoshi M, Tsuneyoshi M, et al. Papillary cystic tumors of the pancreas. Assessment of their malignant potential. Cancer 1993;71:82-92. 